\title{
Caregivers' joint depressive symptoms and preschoolers' daily routines in Chinese three-generation families: Does household chaos matter?
}

\author{
Yongqiang Jiang ${ }^{1} \cdot$ Ting $\mathrm{He}^{1} \cdot$ Xiuyun $\mathrm{Lin}^{1}\left(\mathbb{D} \cdot \mathrm{Qing}_{\mathrm{Zhou}}{ }^{2} \cdot\right.$ Qinglu Wu $^{3}$ \\ Accepted: 5 March 2021 / Published online: 17 April 2021 \\ (C) The Author(s), under exclusive licence to Springer Science+Business Media, LLC, part of Springer Nature 2021
}

\begin{abstract}
The benefits of routines for children have been consistently demonstrated in previous literature. However, factors that may confer risks for child routines have seldom been examined, particularly in families where parents and grandparents co-care the children. This study aimed to investigate the associations of parents' and grandparents' depressive symptoms with preschoolers' daily routines in Chinese three-generation families and to determine whether household chaos mediated or moderated the associations. The participants were from 171 urban three-generation families where mothers, fathers, and grandmothers (97 paternal and 74 maternal) were primary caregivers. Mothers, fathers, and grandmothers reported their depressive symptoms at Wave 1; at Wave 2 (during the COVID-19 pandemic), caregivers reported household chaos and child routines. The results revealed that child routines were negatively predicted by parents' joint depressive symptoms rather than grandmothers' depressive symptoms. In the associations, household chaos acted as a mediator rather than a moderator. Specifically, household chaos marginally mediated the associations between parents' and grandmothers' depressive symptoms and child routines only in maternal three-generation families. These findings suggest that in three-generation families, caregivers with more depressive symptoms may elicit more chaotic family environments, which may in turn compromise their children's daily routines.
\end{abstract}

Keywords Child routines $\cdot$ Depressive symptoms $\cdot$ Household chaos $\cdot$ Three-generation families

Activities that occur in "the same order and at about the same time each day" are defined as routines (Cassidy, 1992, p. 52). Children thrive on routines because routines provide structure, boundaries, and order in children's daily lives and help children establish a sense of predictability and controllability (Spagnola \& Fiese, 2007). Many researchers have demonstrated that routines facilitate the socialization of self-regulation, promote physical health and socioemotional adjustment, and reduce behavioral problems in young children (e.g., Bater \& Jordan, 2017; Ferretti \& Bub, 2014; Lanza \& Drabick, 2011). Given the significance of routines in childhood, understanding

Xiuyun Lin

linxy@bnu.edu.cn

1 Institute of Developmental Psychology, Faculty of Psychology, Beijing Normal University, Beijing 100875, China

2 Department of Psychology, University of California, Berkeley, CA 94720, USA

3 Department of Psychology, Faculty of Social Sciences, University of Macau, Macau, China factors that contribute to setting routines for children is important because those factors could be targeted as points of intervention.

Three terms revolving around routines have been well studied. Family routines, pertaining to the entire family unit, refer to the level at which family members create structure and organization in the home (Boyce et al., 1983). Child routines are more specific than family routines. It refers to repetitive behaviors, such as play, reading, meal, sleep, and being disciplined, in the daily and/or weekly life of a specific child (Sytsma et al., 2001; Wittig, 2005). Bedtime routines are even more specific, related to a child's sleep (Henderson \& Jordan, 2010; Mindell et al., 2015). Compared with family routines, child routines and bedtime routines function as proximal and general setting events for child development (Mindell et al., 2015; Sytsma et al., 2001).

This study explored preschoolers' daily routines in Chinese three-generation families. Setting routines might be challenging in such families. More than half of Chinese children under the age of 6 in urban areas are co-cared for by their parents and grandparents, especially grandmothers (Yue, 2018). In such families, it is likely to be crowded and chaotic, which may 
entail disadvantages for child routines (Larsen \& Jordan, 2020). On the other hand, according to Greenfield's (2009) multilevel theory of social change and human development, parents and grandparents growing at different ages are likely to have different values or attitudes on living and parenting (Zhou et al., 2018). This commonly leads to both cooperation and disagreements on childrearing (e.g., living, leisure, and discipline) between parents and grandparents (e.g., Goh \& Kuczynski, 2010; Xiao, 2016). In such an intergenerational co-caring relationship, child routines are one of the intersections and indicators. When parents and/or grandparents have depressive symptoms, establishing and keeping routines for children may be difficult (Spagnola \& Fiese, 2007). This study was designed to examine the associations of parents' and grandparents' depressive symptoms with preschoolers' routines and whether household chaos matters in such associations.

\section{Caregivers' Depressive Symptoms and Child Routines}

When children experience positive interactions with their parents, they may be apt to have daily routines; thus, child routines could be understood as the extension or outcomes of parental involvement or parenting practices (Bater \& Jordan, 2017; Ren et al., 2019; Wittig, 2005). The factors that favor or impede parental effectiveness may also contribute to child routines. Among them, parental depressive symptoms deserve much attention (Goodman, 2007). Depressive symptoms are characterized by intense negative affection, infrequent positive affection, motivational declines, social-cognitive biases, social withdrawing behaviors, etc. Due to motivational declines or affective unavailability, a depressed parent may have difficulty of accessing the agency or energy for engaging in consistent interactions with the child (such as reading bedtime stories). Depressed parents also tend to generate self-oriented goals rather than children-oriented goals (e.g., Dix et al., 2004), leading to low structure in children's daily activities. Even if attempted, those interactions may fall short of pleasure; children may be unwilling to participate regularly. At the couple level, for partners with more depressive symptoms, it is challenging to navigate the ups and downs of their coparenting relationship, which further undermines child routines (e.g., Ren \& Xu, 2019). However, the negativity of parental depressive symptoms on child routines has not been extensively examined, and in the limited studies, only mothers' depressive symptoms were concerned (Churchill \& Stoneman, 2004; Manczak et al., 2017; McLoyd et al., 2008).

As research progressed, fathers' depressive symptoms, as well as other mental health problems, have received increasing attention as determinants of child outcomes. Meta-analysis reports have consistently declared the relation between fathers' depressive symptoms and child behavioral problems, although the effect size is weaker than that of the relation between mothers' depressive symptoms and child outcomes (Cheung \& Theule, 2019; Connell \& Goodman, 2002; Sweeney \& MacBeth, 2016). Therefore, in the average nuclear families, it is rational to consider that mothers' and fathers' depressive symptoms work together to confer environmental risks for child outcomes (Natsuaki et al., 2014).

In Chinese three-generation families, the involvement of grandparents, especially grandmothers, makes the case more complicated than that in nuclear families. Different from the common norm of grandparental involvement in Western families, "being there but not interfering" (Mason et al., 2007), the norm in Chinese families is "being there and interfering" (Chen et al., 2011). Grandparents substantially share part of childrearing issues and reduce parental stress (e.g., Li \& Liu, 2019). The co-caring relationship between parents and grandparents is called "the intergenerational parenting coalition" (Goh \& Kuczynski, 2010). According to the family system theory (Minuchin, 1985), in such families, grandparents' psychosocial maladjustment may also contribute to making family functioning challenging. For example, Zou et al. (2019) found that grandmothers' depressive symptoms contributed to either their own or the mothers' stress and dysfunctional interactions with children. Thus, in Chinese three-generation families, grandparents' depressive symptoms might, together with parents' depressive symptoms, confer environmental risks for child routines.

\section{Household Chaos Matters}

In the relation between caregivers' depressive symptoms and child routines, household chaos may matter. Chaotic household environments lack structure, order, and predictability, and are characterized by high levels of noise, crowing, instability, and disorganization (Matheny et al., 1995). Living in such chaotic home, children's daily routines may not be guaranteed (Larsen \& Jordan, 2020). High levels of unpredictability may confuse children's understanding of the connection between stimuli and feedback and interfere with children's internalization of rules, which progressively impede child socioemotional adjustment (Evans \& Wachs, 2010). Empirically, the detrimental effect of household chaos on child outcomes (e.g., cognitive development, self-regulation, academic achievement, and socioemotional adjustment) has been established in extensive research, even when family income or socioeconomic status is controlled for (Wachs \& Evans, 2010).

Depressed parents may be inferior in cognitive and affective function so that they are less effective in maintaining familial structure and order (e.g., Bridgettet al., 2013; Brieant et al., 2017). Particularly, their emotional unavailability and negativity easily cause family daily hassles. Such hassles are chaotic 
per se, and may also further interfere with their motivations and abilities to maintain an organized and stable family environment (e.g., Wang et al., 2013). Thus, household chaos may work as a mediator accounting for the relation between caregivers' depressive symptoms and child routines. Household chaos may also interfere with caregivers' cognitive and emotional regulatory competences, leading to less adaptive parenting practices (Brieant et al., 2017; Deater-Deckard et al., 2012). Subsequently, caregivers' efforts to establish and keep routines for children may be compromised. The case might be heavier for caregivers with higher levels of depressive symptoms. Thus, household chaos may intensify the link from caregivers' depressive symptoms to child routines.

Hur et al. (2015) simultaneously examined the mediating and moderating roles of household chaos in the link between parental depressive symptoms and child outcomes. Only the mediation hypothesis was supported. However, in the study, only a single parent's depressive symptoms were accessed, where respondents were primarily mothers $(88.89 \%)$, fathers (8.8\%), and grandparents (2.08\%). Accessing only one caregiver's influence less closely approximates children's lived experiences (Natsuaki et al., 2014). Thus, the findings in Hur et al.'s (2015) study provide limited references for understanding the role of household chaos in the association between caregivers' depressive symptoms and child routines in Chinese three-generation families.

\section{The Present Study}

The current study had three aims. (1) We examined the associations between parents' and grandmothers' depressive symptoms and child routines in Chinese three-generation families. We expected that in families where parents and grandparents had higher levels of depressive symptoms, children had less routines in daily lives. (2) The mediating and moderating roles of household chaos in the associations above were examined. We preferred to expect that it was mediational rather than conditional. It might be that parents and grandparents with higher levels of depressive symptoms would provide more chaotic family environments, in which child routines were compromised. (3) We also examined whether there were differences in the associations above between paternal and maternal three-generation families. Because this question was exploratory, no specific hypothesis was made.

\section{Method}

\section{Participants}

The participants were preschoolers, mothers, fathers, and grandmothers from 171 urban three-generation families.
Half of the preschoolers were girls $(n=86)$, and the average age was 49.39 months old ( $S D=7.36$, range from 39 to 63 months). More than half were reported as the only-child $(62.6 \%)$ during this investigation. The mean age of mothers and fathers was $34.59(S D=3.24)$ and $36.40(S D=4.52)$ years old, respectively. More than $95 \%$ of the mothers and fathers had a college degree or higher. More than half of the grandmothers were paternal $(n=97)$ and native $(n=99)$. The average age of the grandmothers was 60.79 years old $(S D=5.08)$. More detailed demographic information is shown in Table 1. These families were recruited from kindergartens in Beijing urban areas, in November and December 2019. Beijing is the capital of China. In 2019, the per capita disposable income of the urban residents was 73,848 RMB (approximately US $\$ 11,298)$.

\section{Procedure}

With the assistance and support of the directors of four public kindergartens, we attempted to invite all the families $(N=$ 1106) to participate in the grandparenting and child development program. Via the teachers, we sent 900 packages to potential families. All the families were asked to return the packages within seven days to their children's teachers. The teachers then sent the completed packages to the research team. In the package, each family would receive an explanation of the study's objectives, a consent form, a copy of child outcomes and family demographic information, a mother's

Table 1 Demographic Information of the Sample $(N=171)$

\begin{tabular}{|c|c|c|}
\hline & \multicolumn{2}{|c|}{ Mean $(S D)$ or $\%(n)$} \\
\hline \multicolumn{3}{|l|}{ Child's } \\
\hline $\operatorname{Sex}(0=$ =boy", 1 ="girl”) & $50.3 \%(86)$ & \\
\hline Age (Months) & $49.39(7.36)$ & \\
\hline Only-child (0=“no", 1 =“yes") & $62.6 \%(107)$ & \\
\hline Parents' & Mother's & Father's \\
\hline Age (years) & $34.59(3.24)$ & $36.40(4.52)$ \\
\hline \multicolumn{3}{|l|}{ Education attainment } \\
\hline 1="High school and below" & $2.3 \%(4)$ & $4.7 \%(8)$ \\
\hline $2="$ College or Bachelor degree" & $71.3 \%(122)$ & $72.4 \%(123)$ \\
\hline $3="$ Master degree and above" & $26.3 \%(45)$ & $22.8 \%(39)$ \\
\hline \multicolumn{3}{|l|}{ Grandmother's } \\
\hline Role (1 =“"paternal", 2 ="maternal") & $56.7 \%(97)$ & \\
\hline Native (0="no", 1="yes") * & $57.9 \%(99)$ & \\
\hline Age (years) & $60.79(5.08)$ & \\
\hline \multicolumn{3}{|l|}{ Education attainment ${ }^{* *}$} \\
\hline $1=$ ="Junior high school and below" & $28.7 \%(49)$ & \\
\hline $2=$ "Technical (high) school" & $39.8 \%(68)$ & \\
\hline $3="$ "College and above" & $22.8 \%(39)$ & \\
\hline
\end{tabular}


questionnaire, a father's questionnaire, and a grandmother's questionnaire. Mothers were asked to complete the copy of child outcomes and family demographic information, and the other copies were to be completed by the corresponding agents alone. We received responses from 695 families.

In the questionnaire of child outcomes and family demographic information, a form was used to reveal the division of parenting responsibilities regarding the target child during the past year. In the form, mothers were asked to report the quantitative extent of the involvement of each potential caregiver, i.e., mother, father, paternal grandmother, paternal grandfather, maternal grandmother, maternal grandfather, and others (e.g., babysitter), in child-rearing tasks (living, leisure, and education) for the target children during the past year. The proportion of caregivers' joint involvement in living, leisure, and education was summed up to $100 \%$, respectively. For example, in a family, the proportion of mother's, father's, paternal grandmother's, paternal grandfather's, maternal grandmother's, maternal grandfather's, and others' (e.g., baby-sitter) care of a child's education was 35\%, 30\%, 20\%, $10 \%, 5 \%, 0 \%$, and $0 \%$, respectively. The forms from 652 families were eligible. Using latent profile analysis (Berlin et al., 2014), 405 families were identified as having an intergenerational co-caring pattern $(62.1 \%)$. Among such families, 283 families $(69.9 \%)$ were included, in which mothers, fathers, and grandmothers completed their own questionnaires.

About six months later (i.e., May 2020), these families were re-recruited via a Chinese survey website to complete an online questionnaire. In this wave, 193 families were retained. The majority of informants were mothers $(87.6 \%)$. Comparisons between families in Wave 1 only and those in both Wave 1 and Wave 2 revealed that mothers and grandmothers in former had reported higher levels of depressive symptoms $\left(t_{\text {mother }}=2.11, p=.036 ; t_{\text {grandmother }}=2.45\right.$, $p=.015)$. In the families which participated in both Wave 1 and Wave 2, 22 families had experienced caregivers' adjustment (e.g., paternal grandmother replaced maternal grandmother) in 2020. Therefore, we obtained a final sample of 171 families, including 97 paternal three-generation families and 74 maternal three-generation families.

\section{Measure}

\section{Depressive Symptoms}

Depressive symptoms were assessed using the 20-item Center for Epidemiologic Studies Depression Scale (CES-D; Radloff, 1977). The CES-D is shown to be a reliable and valid measurement for Chinese people (e.g., Han \& Jia, 2012). Caregivers were asked to rate each item from 1 (less than a day) to 4 ( 5 to 7 days of the past week). Scores were summed up for all items, with higher scores indicating higher levels of depressive symptoms. In this study, the Cronbach's $\alpha$ values of mothers', fathers, and grandmothers' reports were $.82, .85$, and .88 , respectively.

\section{Household Chaos}

The Confusion, Hubbub, and Order Scale (CHAOS; Matheny et al., 1995) was used to measure household chaos in Wave 2. The scale consists of 15 phrases, capturing the level of confusion and disorganization in the home (e.g., "We almost always seem to be rushed"). Participants rated each item on a 5-point scale from 1 (not like my family at all) to 5 (like my family very much). An overall score was calculated by summing the scores for all 15 items. This scale has been validated in Chinese families (Chang et al., 2016). In this study, the scale showed acceptable internal consistency, with a Cronbach's $\alpha$ value of .83 .

\section{Daily Routines}

Two measures were used to assess preschoolers' daily routines in Wave 2. One was the Chinese version of the Child Routine questionnaire: Preschool (Ren \& Fan, 2019; Wittig, 2005). The original version of this scale has 35 items (Wittig, 2005). Ren and Fan (2019) adapted it in Chinese preschoolers and retained 21 items. The daily living subscale ( 8 items) focuses on preschoolers' consistency of routines in daily living activities, such as mealtime or bedtime. The activities subscale ( 8 items) measures children's routines of involvement in social or educational activities, such as cleaning-up and reading. The discipline subscale ( 5 items) captures the extent to which children are consistently disciplined by caregivers. The other one was the Consistency subscale from the Bedtime routines Questionnaire (Henderson \& Jordan, 2010). The subscale has 10 items. Participants reported their child's five sleep routines (i.e., performing the same activities before going to bed, performing bedtime activities in the same order, sleeping in the same place, going to bed at the same time, and getting put to bed by the same person) during week-nights and weekends for the past month. Caregivers reported the frequency of daily routines on a 5-point Likert scale ranging from 1 (almost never) to 5 (almost always). A mean routine score was calculated by averaging all the items of each subscale, with higher scores indicating more routines. In this study, the Cronbach's $\alpha$ values of living routine, activity routine, discipline routine, and sleep routine were $.80, .82, .77$, and .87 .

\section{Analytical Plan}

Preliminary analyses were conducted within SPSS 26.0. We first created a combined estimate of parents' joint depressive symptoms in a family using the mean values of the standardized z-scores of mothers' and fathers' depressive symptoms. Correlations analyses among study variables were then 
conducted. We also conducted correlation analyses between outcome variables (i.e., household chaos and daily routines) and demographic variables (i.e., child gender, child age, only child, parental and grandmothers' age and educational attainment) to identify any necessary control variables for the primary analyses. Then, as Ren and Xu (2019) did, we constructed child routines as a latent variable with living routine, activity routine, discipline routine, and sleep routine as indicators. Mplus 7.0 (Muthén \& Muthén, 1998-2012) was used to perform the primary analyses. Model fit was assessed using the following indices: non-significant $\chi^{2}$ value, a root mean square error of approximation (RMSEA) and a standardized root mean square residual (SRMR) less than 0.08 , and a comparative fit index (CFI) and a Tucker-Lewis index (TLI) over 0.90 (Kline, 2011). Indirect effects were tested using the bootstrap strategy with 5000 iterations. The $95 \%$ confidence interval $(\mathrm{CI})$ excluding 0 indicates the significance of any given meditational pathway. Next, whether household chaos worked as a moderator was examined. Within SPSS, we first centered the values of all study variables and then calculated the interaction terms between parents' joint and grandmothers' depressive symptoms and household chaos. The moderation hypotheses were also examined within Mplus 7.0.

\section{Results}

\section{Preliminary Analyses}

Descriptive statistics and the correlations for the main study variables are displayed in Table 2. In paternal three-generation families, caregivers' depressive symptoms tended to be related to household chaos rather than child routines, whereas in maternal three-generation families, caregivers' depressive symptoms tended to be related to child routines rather than household chaos. The associations between household chaos and child routines were more significant in paternal three-generation families than those in maternal three-generation families. We also conducted correlation analyses between outcome variables and demographic variables. As shown in Table 3, children's age, mothers' education attainment and grandmothers' age and education attainment had (marginally) significant associations with living routine and activity routine. Therefore, these variables would be included as covariates in the primary analyses.

\section{Primary Analyses}

First, the model of caregivers' depressive symptoms predicting child routines fitted the data well: $\chi^{2}(19)=27.94$, $p=.085, \mathrm{CFI}=.961, \mathrm{TLI}=.943, \mathrm{RMSEA}=.074(90 \% \mathrm{CI}$ $[.000, .129])$, SRMR $=.045$. In both paternal and maternal three-generation families, parents' joint depressive symptoms were negatively associated with child routines (for paternal, $\beta=-.22, p=.043$; for maternal, $\beta=-.36, p=.001$ ), while grandmothers' depressive symptoms were non-significantly associated with child routines (for paternal, $\beta=-.13$, $p=.275$; for maternal, $\beta=-.18, p=.130$ ). We separately compared the predicting effect of parents' joint and grandmothers' depressive symptoms between the paternal and maternal three-generation families, and no significant difference was found $\left(\Delta \chi^{2} \mathrm{~s}(1)<1.53, p \mathrm{~s}>.215\right)$.

Then, we added household chaos into the model. The mediation model also fitted the data well: $\chi^{2}(25)=33.61$, $p=.117, \mathrm{CFI}=.968, \mathrm{TLI}=.948, \mathrm{RMSEA}=.063(90 \% \mathrm{CI}$ $[.000, .114])$, SRMR $=.065$. The results are displayed in Fig. 1. In paternal three-generation families, parents' joint depressive symptoms $(\beta=.30, p=.001)$ and grandmothers' depressive symptoms $(\beta=.30, p=.001)$ significantly predicted household chaos, which in turn was marginally significantly associated with child routines $(\beta=-.23, p=.060)$. The

Table 2 Means, standard deviations, and correlations among the key study variables

\begin{tabular}{|c|c|c|c|c|c|c|c|c|c|c|c|}
\hline & 1 & 2 & 3 & 4 & 5 & 6 & 7 & 8 & 9 & Mean & $S D$ \\
\hline \multicolumn{12}{|l|}{ Wave 1} \\
\hline $1 \mathrm{M}$ depressive symptoms & - & $.28^{*}$ & $.80^{* * * *}$ & .08 & $.20^{+}$ & -.10 & -.16 & -.11 & -.16 & 17.47 & 7.08 \\
\hline $2 \mathrm{~F}$ depressive symptoms & $.24^{*}$ & - & $.80^{* * * *}$ & -.02 & .17 & $-.31^{* *}$ & $-.23^{*}$ & $-.20^{+}$ & $-.42^{* * * *}$ & 10.14 & 9.11 \\
\hline 3 PJ_depressive symptoms & $.79^{* * *}$ & $.79^{* * * *}$ & - & .04 & $.23^{*}$ & $-.25^{*}$ & $-.25^{*}$ & $-.20^{+}$ & $-.36^{* *}$ & .00 & .80 \\
\hline $4 \mathrm{GM}$ _ depressive symptoms & .05 & $.25^{*}$ & $.18^{+}$ & - & $.23^{*}$ & -.09 & -.12 & -.19 & -.17 & 7.30 & 6.18 \\
\hline \multicolumn{12}{|l|}{ Wave $2^{-}$} \\
\hline 5 Household Chaos & $.25^{*}$ & $.32^{* * * *}$ & $.36^{* * * *}$ & $.35^{* * *}$ & - & $-.32^{* *}$ & $-.42^{* * * *}$ & $-.27^{*}$ & $-.36^{* *}$ & 2.08 & .51 \\
\hline 6 Living routine & -.11 & $-.21^{*}$ & $-.20^{*}$ & $-.18^{+}$ & $-.26^{* *}$ & - & $.53^{* * * * *}$ & $.51^{\text {****** }}$ & $.64^{* * * * *}$ & 4.67 & .39 \\
\hline 7 Activity routine & -.16 & -.15 & $-.20^{+}$ & -.06 & $-.27^{* *}$ & $.53^{* * * *}$ & - & $.59^{\text {******* }}$ & $.54^{* * * * *}$ & 4.10 & .62 \\
\hline 8 Discipline routine & -.05 & $-.26^{* *}$ & $-.20^{+}$ & $-.26^{*}$ & $-.17^{+}$ & $.25^{*}$ & $.60^{* * * *}$ & - & $.61^{* * * *}$ & 4.33 & .51 \\
\hline 9 Sleep routine & -.11 & -.05 & -.11 & $-.19^{+}$ & $-.22^{*}$ & $.40^{* * *}$ & $.49^{* * * *}$ & $.38^{* * * *}$ & - & 4.62 & .45 \\
\hline Mean & 18.16 & 10.94 & .00 & 9.94 & 1.99 & 4.70 & 4.11 & 4.24 & 4.62 & - & - \\
\hline$S D$ & 6.67 & 8.43 & .79 & 7.60 & .52 & .39 & .54 & .55 & .40 & - & - \\
\hline
\end{tabular}

Correlations in bottom of diagonal are from paternal three-generation families $(n=97)$, in top are from maternal three-generation families $(n=74) ; M_{-}$ mothers', $F_{-}$fathers', $P J_{-}$parents' joint, $G M_{-}$grandmothers' ${ }^{+} p<.10,{ }^{*} p<.050,{ }^{* *} p<.010,{ }^{* * *} p<.001$ 
Table 3 Correlations between outcome and demographic variables

\begin{tabular}{|c|c|c|c|c|c|}
\hline & Household Chaos & Living routine & Activity routine & Discipline routine & Bedtime routine \\
\hline \multicolumn{6}{|l|}{ Child's } \\
\hline Sex & .08 & -.04 & -.04 & .00 & -.06 \\
\hline Age & .05 & $.16^{*}$ & -.12 & -.07 & .03 \\
\hline Only child & -.03 & -.05 & .12 & .05 & -.02 \\
\hline \multicolumn{6}{|l|}{ Mother's } \\
\hline Age & .06 & .00 & .07 & .02 & -.03 \\
\hline Education attainment & -.06 & $-.16^{*}$ & -.03 & $-.17^{*}$ & -.02 \\
\hline \multicolumn{6}{|l|}{ Father's } \\
\hline Age & .10 & .03 & .01 & -.01 & .01 \\
\hline Education attainment & -.08 & -.02 & .12 & -.07 & .08 \\
\hline \multicolumn{6}{|l|}{ Grandmother's } \\
\hline Native & .06 & .09 & -.10 & .07 & .07 \\
\hline Age & .07 & .12 & $.14^{+}$ & .13 & .02 \\
\hline Education attainment & -.01 & .01 & $.15^{+}$ & .04 & .07 \\
\hline
\end{tabular}

$+p<.10{ }^{*} p<.050$

indirect effects of parents' joint depressive symptoms $[\beta=$ $-.07,95 \% \mathrm{CI}=(-.17, .03), p=.174]$ and grandmothers' depressive symptoms $[\beta=-.07,95 \% \mathrm{CI}=(-.17, .03), p=.183]$ via household chaos on child routines were neither significant. The direct links from parents' joint depressive symptoms $(\beta=$ $-.15, p=.174)$ and grandmothers' depressive symptoms $(\beta=$ $-.05, p=.664)$ to child routines were also non-significant. The model explained $11.5 \%$ of the variance in child routines and $21.2 \%$ of the variance in household chaos. In maternal threegeneration families, parents' joint depressive symptoms $(\beta=.22, p=.040)$ and grandmothers' depressive symptoms $(\beta=.22, p=.039)$ significantly predicted household chaos, which in turn was significantly associated with child routines $(\beta=-.37, p=.001)$. The direct link from parents' joint depressive symptoms to child routines was significant $(\beta=-.27$, $p=.015)$, but from grandmothers' depressive symptoms was not $(\beta=-.09, p=.442)$. The indirect effects of parents' joint depressive symptoms $[\beta=-.09,95 \% \mathrm{CI}=(-.17, .01)$, $p=.079]$ and grandmothers' depressive symptoms $[\beta=-.09$, $95 \% \mathrm{CI}=(-.17, .02), p=.098]$ via household chaos on child routines were both marginally significant. The model explained $28.2 \%$ of the variance in child routines and $10.2 \%$ of the variance in household chaos. We also separately compared the predicting effects from parents' joint and grandmothers' depressive symptoms to household chaos and then to child routines between the paternal and maternal threegeneration families, and no significant difference was found $\left(\Delta \chi^{2} \mathrm{~s}(1)<1.60, p \mathrm{~s}>.207\right)$.

Next, we treated household chaos as a potential moderator. The results revealed that in both paternal and maternal threegeneration families, the interaction terms were not significant in predicting child routines $(|\beta| \mathrm{s}<.16, p \mathrm{~s}>.186)$. It meant that the moderation hypothesis was not supported.

\section{Discussion}

Routines benefit children. With a sample of Chinese threegeneration families where mothers, fathers, and grandmothers were primary caregivers, the present study was the first to demonstrate that caregivers' depressive symptoms were negatively associated with child routines. In such a link, it was

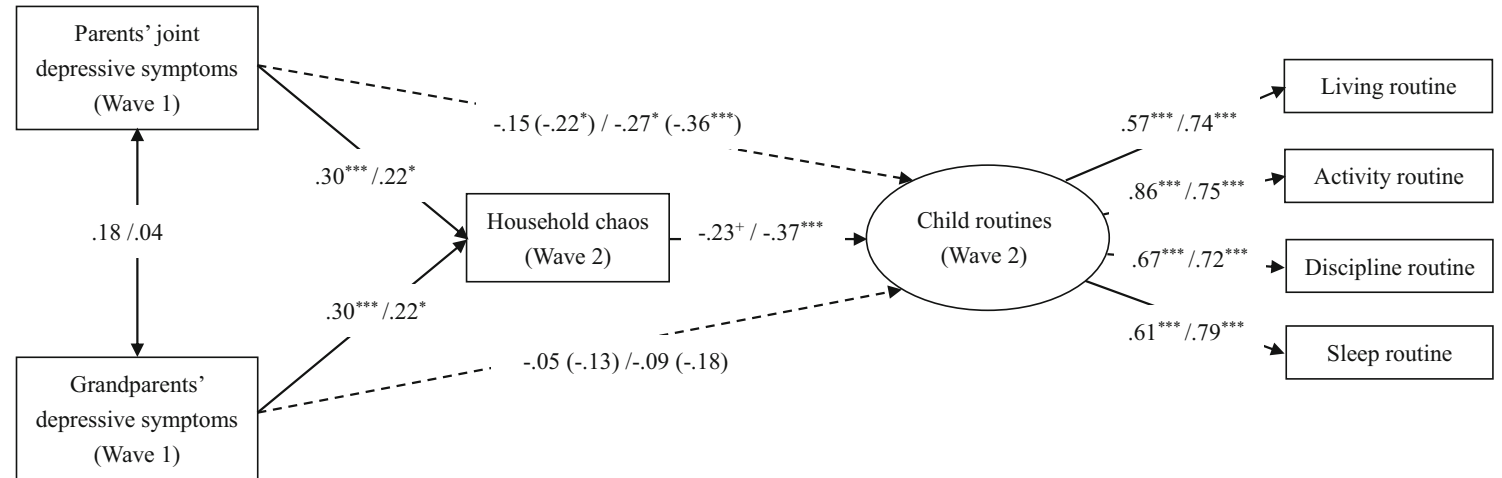

Fig. 1 Mediation model. Note. Children's age and mothers' educational attainment were included as covariates but not shown. Coefficients at left side of the slash are from paternal three-generation families, coefficients at the right side are from maternal three-generation families. Coefficients in brackets are from the model without including the mediator. . ${ }^{+} p<.10$, ${ }^{*} p<.050,{ }^{* *} p<.010,{ }^{* * *} p<.001$ 
found that household chaos worked only as a mediator. The findings preliminarily advance our understanding of the associations between caregivers' depressive symptoms, household chaos, and child routines in Chinese three-generation families.

The first contribution of this study is that in three-generation families where caregivers have higher levels of depressive symptoms, children have less routines in their daily lives. This finding is in line with and extends previous studies in which only the negativity of mothers' depressive symptoms on child routines was considered (Churchill \& Stoneman, 2004; Manczak et al., 2017; McLoyd et al., 2008). More importantly, we found that the negativity of caregivers' depressive symptoms on child routines were from parents rather than grandmothers. In Chinese three-generation families, it is common for grandparents, especially grandmothers, to regularly help adult children (i.e., mothers and fathers) take care of their grandchildren. In such families, these caregivers establish an "intergenerational parenting coalition", where cooperation and conflicts are both commonplace (e.g., Goh \& Kuczynski, 2010). Each caregiver's involvement and parenting effectiveness contribute to child routines. When a mother has more depressive symptoms, she may tend to follow her own selforiented goals when taking care of the grandchild (e.g., Dix et al., 2004), which may elicit disagreements with father or grandmother, leading to low structure in child's routines (Ren $\& \mathrm{Xu}, 2019)$. In this study, child routines were assessed in May 2020 when was during the COVID-19 pandemic. During the hard period, parents might have more time to take care their children who had to stay at home rather than kindergartens (Prime et al., 2020). Parents might become more primary than grandmothers in regulating preschoolers' daily lives.

Another contribution is that household chaos helped partially account for the association between caregivers' depressive symptoms and child routines. In three-generation families where all caregivers have high levels of depressive symptoms, it is challenging to maintain organized and stable family environments. This finding is partially in line with Hurs et al.'s (Hur et al., 2015) study, evidencing the negative effects of depressive symptoms on household chaos. It might be that depressive symptoms increase a family's daily hassles in addressing domestic affairs. For example, a depressed mother may have many objections towards fathers' and grandmothers' behaviors. A depressed grandmother may frequently argue against parents' arrangements. Such displeasure will impede the familial structure and order, leading to a chaotic family environment. In such a context, child routines are unlikely to be guaranteed (Larsen \& Jordan, 2020). It is noteworthy that the mediating role of household chaos in the associations between caregivers' depressive symptoms and child routines was weak. It may be that during the pandemic preschoolers had to stay at home, which brought other ways undermining child daily routines. We also examined whether household chaos exacerbated the negative effects of depressive symptoms on child routines. However, the possibility was not supported.

The third contribution is that the differences between paternal and maternal three-generation families in the associations among caregivers' depressive symptoms, household chaos, and child routines were preliminarily revealed. Specifically, household chaos played a mediating role in the associations between parents' and grandmothers' depressive symptoms and child routines in maternal but not paternal three-generation families. It may be that parents and grandmothers tie more closely in maternal than paternal three-generation families (e.g., Li et al., 2016). Parents or grandmothers' individual psychosocial maladjustment easily cross-over to the interactions between caregivers and children (Minuchin, 1985). Such close intergenerational coparenting interactions in maternal three-generation families drive the significant links from caregivers' depressive symptoms to household chaos and child routines. However, in paternal three-generation families, caregivers' depressive symptoms only contributed to chaotic household environment. Although not considered in this study, it is reasonable to believe that intergenerational coparenting relationship may help explain the negative effects of caregivers' depressive symptoms on household chaos and child routines, which are highly encouraged to be examined in future research.

Our findings should be interpreted with caution since this study has several limitations. First, household chaos and child routines were obtained from same informants' reports, which may inflate the magnitude of the associations due to the shared method variance. Future work that includes multiple methods, such as self-reports, observation, or interviews, will be beneficial. Second, families' participation in our program was voluntary, so some dysfunctional families or individuals with high psychosocial maladjustment might not be included. And, some families where mothers and grandmothers had high levels of depressive symptoms did not participate in Wave 2. In this case, the current findings may have been biased. Third, our questionnaires were asked to be completed by the corresponding agents alone at home. The possibility that people discussed the questionnaire in their families could not be ruled out. Finally, the current sample was from Beijing. In Beijing, the average family's socioeconomic status and adults' educational attainment are almost the highest in the nation. Our sampling may limit the generalizability of our findings to other cities and rural areas.

Despite these limitations, this study has a notable strength. In previous studies about the co-caring relationship of parents and grandparents in Chinese three-generation families (e.g., Li et al., 2020), the families were screened by asking whether the grandparents participated in the raising of the grandchildren (yes/no). The screening method does not access the extent of each potential caregiver's involvement and thus cannot identify which caregiver(s) are primary. Instead, in the present study, by asking and analyzing the quantitative extent to 
which each potential caregiver, i.e., mother, father, paternal grandmother, paternal grandfather, maternal grandmother, maternal grandfather, and others (e.g., babysitter), was involved child-rearing tasks (living, leisure, and education) for the target children during the past year, we could statistically make sure that mothers, fathers, and grandmothers were the primary caregivers in our samples of three-generation families. This step is of much value. Future research is highly encouraged to refer to the screening method.

Although preliminary, our findings suggest the significance of targeting caregivers' socioemotional maladjustment problems and household chaos for enhancing preschoolers' daily routines. First, intervention or psychoeducation programs that consist of the importance and the elements of child routines should point out that caregivers' depressive symptoms and household chaos are not irrelevant. Second, family practitioners or therapists should be mindful of the joint effect of depressive symptoms across caregivers. Third, to alleviate the negative effects of caregivers' depressive symptoms on child routines, family practitioners or therapists dealing with caregivers' depressive symptoms can also be encouraged to teach or help families create and maintain quiet and organized households.

Acknowledgments The study described in this report was in part funded by 2018 National Natural Science Foundation of China (Grand No. 31800935) and 2017 Chau Hoi Shuen Foundation Women in Science Research Grant. The funders had no role in study design, data collection and analysis, decision to publish, or preparation of the manuscript. We are appreciative of the grandparents, parents, children, and kindergartens who participated in our study and the many people who assisted in data collection.

Data Availability The data that support the findings of this study are available from the corresponding author upon reasonable request.

\section{Declarations}

All procedures performed in studies involving human participants were in accordance with the ethical standards of the institutional research committee and with the 1964 Helsinki Declaration and its later amendments or comparable ethical standards.

Conflict of Interest The authors declare that they have no known competing financial interests or personal relationships that could have appeared to influence the work reported in this paper.

\section{References}

Bater, L. R., \& Jordan, S. S. (2017). Child routines and self-regulation serially mediate parenting practices and externalizing problems in preschool children. Child \& Youth Care Forum, 46, 243-259. https://doi.org/10.1007/s10566-016-9377-7.

Berlin, K. S., Williams, N. A., \& Parra, G. R. (2014). An introduction to latent variable mixture modeling (part 1): Overview and crosssectional latent class and latent profile analyses. Journal of Pediatric Psychology, 39, 174-187. https://doi.org/10.1093/jpepsy/jst084.
Boyce, W. T., Jensen, E. W., James, S. A., \& Peacock, J. L. (1983). The family routines inventory: Theoretical origins. Social Science \& Medicine, 17, 193-200. https://doi.org/10.1016/0277-9536(83)90116-8.

Bridgett, D. J., Burt, N. M., Laake, L. M., \& Oddi, K. B. (2013). Maternal self-regulation, relationship adjustment, and home chaos: Contributions to infant negative emotionality. Infant Behavior and Development, 36, 534-547. https://doi.org/10.1016/j.infbeh.2013. 04.004.

Brieant, A., Holmes, C. J., Deater-Deckard, K., King-Casa, B., \& KimSpoon, J. (2017). Household chaos as a context for intergenerational transmission of executive functioning. Journal of Adolescence, 58, 40-48. https://doi.org/10.1016/j.adolescence.2017.05.001.

Cassidy, A. (1992). When, why, how to get your baby into a routine. Working Mother, 15(56), 58-60.

Chang, S., Song, Y., \& Guo, H. (2016). Home chaos and migrant children's security: The moderating effect of gender [in Chinese]. Chinese Journal of Special Education, 4, 66-78.

Chen, F., Liu, G., \& Mair, C. A. (2011). Intergenerational ties in context: Grandparents caring for grandchildren in China. Social Forces, 90, 571-594. https://doi.org/10.1093/sf/sor012.

Cheung, K., \& Theule, J. (2019). Paternal depression and child externalizing behaviors: A meta-analysis. Journal of Family Psychology, 33, 98-108. https://doi.org/10.1037/fam0000473.

Churchill, S. L. \& Stoneman, Z. (2004). Correlates of family routines in head start families. Early Childhood Research \& Practice, 6, https:// eric.ed.gov/?id=EJ1084842

Connell, A. M., \& Goodman, S. H. (2002). The association between psychopathology in fathers versus mothers and children's internalizing and externalizing behavior problems: A meta-analysis. Psychological Bulletin, 128, 746-773. https://doi.org/10.1037/ 0033-2909.128.5.746.

Deater-Deckard, K., Chen, N., Wang, Z., \& Bell, M. A. (2012). Socioeconomic risk moderates the link between household chaos and maternal executive function. Journal of Family Psychology, 26, 391-399. https://doi.org/10.1037/a0028331.

Dix, T., Gershoff, E. T., Meunier, L. N., \& Miller, P. C. (2004). The affective structure of supportive parenting: Depressive symptoms, immediate emotions, and child-oriented motivation. Developmental Psychology, 40, 1212-1227. https://doi.org/10.1037/0012-1649.40.6.1212.

Evans, G. W., \& Wachs, T. (Eds.). (2010). Chaos and its influence on children's development: An ecological perspective. Washington, DC: American Psychological Association. https://doi.org/10.1037/ 12057-000

Ferretti, L. K., \& Bub, K. L. (2014). The influence of family routines on the resilience of low-income preschoolers. Journal of Applied Developmental Psychology, 35, 168-180. https://doi.org/10.1016/j. appdev.2014.03.003.

Goh, E. L., \& Kuczynski, L. (2010). 'Only children' and their coalition of parents: Considering grandparents and parents as joint caregivers in urban Xiamen, China. Asian Journal of Social Psychology, 13, 221231. https://doi.org/10.1111/j.1467-839X.2010.01314.x.

Goodman, S. H. (2007). Depression in mothers. Annual Review of Clinical Psychology, 3, 107-135. https://doi.org/10.1146/annurev. clinpsy.3.022806.091401.

Greenfield, P. M. (2009). Linking social change and developmental change: Shifting pathways of human development. Developmental Psychology, 45, 401-418. https://doi.org/10.1037/a0014726.

Han, M., \& Jia, X. (2012). Reliability and validity of Center for Epidemiological Studies Depression Scale in different rural populations [in Chinese]. Chinese Journal of Public Health, 28, 1265-1267.

Henderson, J. A., \& Jordan, S. S. (2010). Development and preliminary evaluation of the bedtime routines questionnaire. Journal of Psychopathology and Behavioral Assessment, 32, 271-280. https://doi.org/10.1007/s10862-009-9143-3.

Hur, E., Buettner, C. K., \& Jeon, L. (2015). Parental depressive symptoms and children's school-readiness: The indirect effect of 
household chaos. Journal of Child and Family Studies, 24, 34623473. https://doi.org/10.1007/s10826-015-0147-1.

Kline, R. B. (2011). Principles and practice of structural equation modeling (3rd ed.), New York: Guilford

Lanza, H., \& Drabick, D. (2011). Family routine moderates the relation between child impulsivity and oppositional defiant disorder symptoms. Journal of Abnormal Child Psychology, 39, 83-94. https:// doi.org/10.1007/s10802-010-9447-5.

Larsen, K. L., \& Jordan, S. S. (2020). Organized chaos: Daily routines link household chaos and child behavior problems. Journal of Child and Family Studies, 29, 1094-1107. https://doi.org/10.1007/ s10826-019-01645-9.

Li, X., \& Liu, Y. (2019). Parent-grandparent coparenting relationship, maternal parenting self-efficacy, and young children's social competence in Chinese urban families. Journal of Child and Family Studies, 28, 1145-1153. https://doi.org/10.1007/s10826-019-01346-3.

Li, X., Xie, J., \& Song, Y. (2016). Grandparents-parents co-parenting and its relationship with maternal parenting stress and children's behavioral problems [in Chinese]. Chinese Journal of Special Education, 4, 71-78

Li, X., Zhou, S., \& Guo, Y. (2020). Bidirectional longitudinal relations between parent-grandparent co-parenting relationships and Chinese children's effortful control during early childhood. Frontiers in Psychology, 11, 152. https://doi.org/10.3389/fpsyg.2020.00152.

Manczak, E. M., Williams, D., \& Chen, E. (2017). The role of family routines in the intergenerational transmission of depressive symptoms between parents and their adolescent children. Journal of Abnormal Child Psychology, 45, 643-656. https://doi.org/10.1007/ s10802-016-0187-z.

Mason, J., May, V., \& Clarke, L. (2007). Ambivalence and the paradoxes of grandparenting. The Sociological Review, 55, 687-706. https:// doi.org/10.1111/j.1467-954X.2007.00748.x.

Matheny, A. P., Wachs, T. D., Ludwig, J. L., \& Phillips, K. (1995). Bringing order out of chaos: Psychometric characteristics of the confusion, hubbub, and order scale. Journal of Applied Developmental Psychology, 16, 429-444. https://doi.org/10.1016/ 0193-3973(95)90028-4.

McLoyd, V. C., Toyokawa, T., \& Kaplan, R. (2008). Work demands, work family conflict, and child adjustment in African American families: The mediating role of family routines. Journal of Family Issues, 29, 1247-1267. https://doi.org/10.1177/0192513X08320189.

Mindell, J. A., Li, A. M., Sadeh, A., Kwon, R., \& Goh, D. Y. (2015). Bedtime routines for young children: A dose-dependent association with sleep outcomes. Sleep, 38, 717-722. https://doi.org/10.5665/ sleep. 4662 .

Minuchin, P. (1985). Families and individual development: Provocations from the field of family therapy. Child Development, 56, 289-302. https://doi.org/10.2307/1129720.

Muthén, L.K., \& Muthén, B.O. (1998-2012). Mplus user's guide (7th ed.). Los Angeles: Muthén \& Muthén

Natsuaki, M. N., Shaw, D. S., Neiderhiser, J. M., Ganiban, J. M., Harold, G. T., Reiss, D., \& Leve, L. D. (2014). Raised by depressed parents: Is it an environmental risk? Clinical Child and Family Psychology Review, 17, 357-367. https://doi.org/10.1007/s10567-014-0169-z.

Prime, H., Wade, M., \& Browne, D. T. (2020). Risk and resilience in family well-being during the COVID-19 pandemic. American Psychologist, 75, 631-643. https://doi.org/10.1037/amp0000660.

Radloff, L.S. (1977). The CES-D scale: A self-report depression scale for research in the general population. Applied Psychological Measurement, 1, 385-401
Ren, L., \& Fan, J. (2019). Chinese preschoolers' daily routine and its associations with parent-child relationships and child self-regulation. International Journal of Behavioral Development, 43, 179184. https://doi.org/10.1177/0165025418811126.

Ren, L., \& Xu, W. (2019). Coparenting and Chinese preschoolers' socialemotional development: Child routines as a mediator. Children and Youth Services Review, 107, 104549. https://doi.org/10.1016/j. childyouth.2019.104549.

Ren, L., Hu, B. Y., \& Song, Z. (2019). Child routines mediate the relationship between parenting and social-emotional development in Chinese children. Children and Youth Services Review, 98, 1-9. https://doi.org/10.1016/j.childyouth.2018.12.016.

Spagnola, M., \& Fiese, B. H. (2007). Family routines and rituals: A context for development in the lives of young children. Infants \& Young Children, 20, 284-299. https://doi.org/10.1097/01.IYC. $0000290352.32170 .5 \mathrm{a}$.

Sweeney, S., \& MacBeth, A. (2016). The effects of paternal depression on child and adolescent outcomes: A systematic review. Journal of Affective Disorders, 205, 44-59. https://doi.org/10.1016/j.jad.2016. 05.073

Sytsma, S. E., Kelley, M. L., \& Wymer, J. H. (2001). Development and validation of the child routines inventory. Journal of Psychopathology and Behavioral Assessment, 23, 241-251. https://doi.org/10.1023/A:1012727419873.

Wachs, T. D., \& Evans, G. W. (2010). Chaos in context. In G. W. Evans \& T. D. Wachs (Eds.), Chaos and its influence on children's development: An ecological perspective (pp. 3-13). Washington, DC: American Psychological Association. https://doi.org/10.1037/12057-001

Wang, Z., Deater-Deckard, K., \& Bell, M. A. (2013). Household chaos moderates the link between maternal attribution bias and parenting. Parenting: Science and Practice, 13, 233-252. https://doi.org/10. 1080/15295192.2013.832569.

Wittig, M. M. (2005). Development and validation of the child routines questionnaire: Preschool. Unpublished doctoral dissertation, Baton Rouge: Louisiana State University

Xiao, S. (2016). Intimate power: The intergenerational cooperation and conflicts in childrearing among urban families in contemporary China. The Journal of Chinese Sociology, 3, 18. https://doi.org/10. 1186/s40711-016-0037-y.

Yue, K. (2018). The intergenerational co-parenting pattern of parents first and grandparents second benefits for grandchildren's well-being: A survey about Chinese grandparental involvement in urban areas [in Chinese]. Children's Study, 1, 3-20

Zhou, C., Yiu, W. Y. V., Wu, M. S., \& Greenfield, P. M. (2018). Perception of cross-generational differences in child behavior and parent socialization: A mixed-method interview study with grandmothers in China. Journal of Cross-Cultural Psychology, 49, 6281. https://doi.org/10.1177/0022022117736029.

Zou, X., Lin, X., Jiang, Y., Xu, J., Qin, S., \& Han, R. Z. (2019). The associations between mothers' and grandmothers' depressive symptoms, parenting stress, and relationship with children: An actorpartner interdependence mediation model. Family Process, 59, 1755-1772. https://doi.org/10.1111/famp.12502.

Publisher's Note Springer Nature remains neutral with regard to jurisdictional claims in published maps and institutional affiliations. 